\title{
Evidence of positive and negative selection associated with DNA methylation
}

\author{
Charlie Hatcher ${ }^{1 *}$, Genetics of DNA Methylation Consortium, Gibran Hemani ${ }^{1}$, Santiago Rodriguez ${ }^{1}$, Tom R. \\ Gaunt $^{1}$, Daniel J. Lawson ${ }^{1,2}$ and Josine L. Min ${ }^{1}$ \\ ${ }^{1}$ MRC Integrative Epidemiology Unit, Population Health Sciences, Bristol Medical School, University of Bristol \\ ${ }^{2}$ Department of Statistical Sciences, School of Mathematics, University of Bristol \\ * Corresponding author: Charlie Hatcher, MRC Integrative Epidemiology Unit, Population Health Sciences, Bristol Medical \\ School, Oakfield House, Oakfield Grove, Bristol BS8 2BN, UK. Email: charlie.hatcher@bristol.ac.uk
}

\begin{abstract}
Signatures of negative selection are pervasive amongst complex traits and diseases. However, it is unclear whether such signatures exist for DNA methylation (DNAm) that has been proposed to have a functional role in disease. We estimate polygenicity, SNP-based heritability and model the joint distribution of effect size and minor allele frequency (MAF) to estimate a selection coefficient $(S)$ for 2000 heritable DNAm sites in 1774 individuals from the Avon Longitudinal Study of Parents and Children. Additionally, we estimate $S$ for meta stable epi alleles and DNAm sites associated with aging and mortality, birthweight and body mass index. Quantification of MAF-dependent genetic architectures estimated from genotype and DNAm reveal evidence of positive $(S>0)$ and negative selection $(S<0)$ and confirm previous evidence of negative selection for birthweight. Evidence of both negative and positive selection highlights the role of DNAm as an intermediary in multiple biological pathways with competing function.
\end{abstract}

\section{Word count: 148}

Keywords: ALSPAC, DNA methylation, natural selection 


\section{Introduction}

Genome-wide association studies (GWASs) have identified many genetic variants (single nucleotide polymorphisms; SNPs) associated with complex traits and diseases ${ }^{1}$. Natural selection plays a role in influencing the genetic architecture of complex traits, altering allele frequency at many genetic loci ${ }^{2}$. Negative selection prevents deleterious mutations from becoming common ${ }^{3}$ and is thought to explain why GWASs have identified many common variants of low effect size ${ }^{4}$. Several studies have shown evidence of negative selection acting on complex traits (including height, body mass index; BMI and birthweight) using the relationship between minor allele frequency (MAF) and SNP effect size to estimate a selection coefficient $(S)^{3,5,6}$. However, there is difficulty in separating the action of selection from genetic drift when using MAF and SNP effect size to characterise genetic architecture ${ }^{7}$.

Most GWA loci reside in non-coding regions and colocalization studies have shown that genetic factors underlying intermediate traits are shared with GWA locis, ${ }^{8,9}$. Intermediate traits such DNA methylation (DNAm) and gene expression may therefore also show signatures of selection. Variation in DNAm can be influenced by age ${ }^{10}$, environmental ${ }^{11}$, genetic ${ }^{12}$ and stochastic ${ }^{13}$ changes. The variability of DNAm maybe caused by natural selection, epigenetic stochasticity ${ }^{14}$ or cellular plasticity ${ }^{15}$. The Genetics of DNA Methylation Consortium (GoDMC) has identified a large number of methylation quantitative trait loci (mQTLs) in blood ${ }^{16}$. They showed that these DNAm sites influenced by genetic factors are polygenic ${ }^{16}$. mQTLs were enriched for a variety of selection metrics (including the singleton density score; SDS ${ }^{17}$ and fixation index; $F_{s t}{ }^{18}$ ) and show a strong negative relationship between MAF and $m Q T L$ effect size ${ }^{19}$. It is therefore likely that natural selection acts on many mQTL variants jointly. However, selection is difficult to detect as DNAm is typically controlled by a local cis variant with large effect size and many physically separated trans variants with small effect sizes. Previous studies on cis-regulatory regions have found evidence of purifying selection on sequence-dependent allelespecific DNAm ${ }^{20}$ and positive selection among African agriculturist populations ${ }^{21}$. Similarly, gene expression traits are polygenic ${ }^{22}$ and SNPs showing signatures of selection are enriched among SNPS associated with gene expression (expression quantitative trait loci; eQTLs) 3 .23.

DNAm has a variety of roles in gene regulation ${ }^{24,25}$, is likely cell type-specific, and can be used as a biomarker for risk stratification and disease detection ${ }^{26,27}$. DNAm at cytosine-guanine dinucleotides (CpGs) has been associated with repression of transcription factor (TF) binding, however, TF binding has also been shown to inhibit DNAm ${ }^{28}$. Across the $450 \mathrm{k}$ sites most commonly measured in epidemiological studies ${ }^{29}$ (which are biased to promoter regions), mean heritability for DNAm has been shown to be around $20 \%{ }^{12}$ and relationships between the heritability of a DNAm site and the number of mQTLs and between heritability and effect size have been found ${ }^{16}$ DNAm sites may have particular properties in terms of natural selection where heritable sites should have increased polygenicity with a larger proportion of SNPs with larger effect sizes ${ }^{4}$. In epigenome-wide association studies (EWASs), DNAm sites have been associated with many complex traits and diseases including those showing signatures of negative selection such as BMl and birthweight ${ }^{3,30,31}$. Additionally, PhenoAge is a composite DNAm predictor of aging (trained on mortality including 42 clinical measures and age), that 
has been predictive of disease risk and mortality ${ }^{32}$. To date, there is little known about whether these sites are a target of selection for example due to antagonistic pleiotropy ${ }^{33}$ where genes required for earlier stages of development may have deleterious effects in later life ${ }^{32}$. Meta stable DNAm sites exhibiting greater similarity than can be explained genetically have also been identified ${ }^{14}$. It may be the case that increased variability of these sites occurred as initial response to the environment before the effect of natural selection.

DNAm may play various roles in underlying biological processes, and therefore we expect it to be subject to both positive and negative selection. Here, we investigate the relationship between MAF and effect size for SNPs at individual DNAm sites from the widely used 450k array to make inferences about the action of natural selection, which we hypothesise may vary for each DNAm site. We utilise BayesNS, a Bayesian mixed linear model method (MLM) that estimates polygenicity, SNP-based heritability and the joint distribution of MAF and effect size ${ }^{3}$. We apply BayesNS to DNAm data from the Accessible Resource for Integrated Epigenomic Studies (ARIES) cohort ${ }^{34}$. 


\section{Results}

\section{Estimation of genetic architecture parameters of DNAm sites}

We used a Bayesian mixed linear model (BayesNS) to estimate genetic architecture parameters of DNAm sites including polygenicity, SNP-based heritability and a selection coefficient $(S)^{3}$. We applied BayesNS to DNAm sites profiled in blood from 1774 mother-offspring individuals from ARIES ${ }^{34}$ and 474,939 independent non-major histocompatibility complex (MHC) and non-lactase (LCT) SNPs. Specifically, we considered 2000 DNAm sites which have 'high' heritability estimates from twin studies $\left(\overline{h_{2}}=89.9 \% \text {, range } 79-99 \% \text {, Table } 1\right)^{12}$, as selection is dependent on a genetic contribution to DNAm variance. Secondly, we analysed 1508 DNAm sites which show non-genetically mediated similarity between monozygotic twins, so-called epigenetic supersimilarity (ESS) DNAm sites ${ }^{14}$. Blood DNA methylation at ESS DNAm sites exhibit plasticity to the periconceptional environment and is associated with risk of cancer. Finally, we considered 513 DNAm sites which combined predict biological age ("PhenoAge"), a trait that is moderately heritable and has been associated with aging, mortality and is predictive of cardiovascular disease risk ${ }^{32}$.

Convergence of the Markov chain Monte Carlo (MCMC) algorithm implies that a single consistent selection signal is found, whilst failure to converge implies that competing, inconsistent sets of SNPs explain the data equally (and poorly). This was assessed with the Raftery-Lewis long-chain diagnostic test $^{35}$ and MCMC trace plots (Figures S1;S2;S3). In line with previous work ${ }^{3}$, DNAm sites which failed convergence checks typically had lower estimates of heritability (Figure S3; Table 1). BayesNS estimates SNP-based heritability and as with the estimates of twin heritability, the highly heritable DNAm sites had the highest mean estimate of SNP based heritability $\left(\overline{h_{S N P}^{2}}=30.2 \%\right.$; SD $=12.4 \%$; Table 1), followed by the ESS DNAm sites $\left(\overline{h_{S N P}^{2}}=26.6 \%\right.$; SD=13.0\%; Table 1) and then the PhenoAge DNAm sites $\left(\overline{h_{S N P}^{2}}=14.8 \%\right.$; SD $=9.7 \%$; Table 1$)$. Since we only consider DNAm sites which passed MCMC convergence diagnostics, (Table 1; Figure S1-S3), these mean estimates are likely higher than we would expect for each set of DNAm sites.

\section{DNAm shows signatures of both positive and negative selection}

BayesNS uses the relationship between SNP effect size and MAF to estimate a selection coefficient $(S)^{3}$. When $S=0$ effect size is independent of MAF and this would reflect a 'neutral' scenario, an $S>$ 0 would represent evidence of positive selection and an $S<0$ would represent evidence of negative selection. Quantification of MAF-dependent genetic architectures revealed the action of both positive $S>0$ and negative $S<0$ selection across all three sets of DNAm sites (Figure 1). On average, estimates are close to zero, (PhenoAge DNAm sites; $\bar{S}=0.04$ ) being mildly negative for the highly heritable $(\bar{S}=-0.15)$ and ESS DNAm sites $(\bar{S}=-0.14)$ (Table 1). Across the distributions we see individual DNAm sites with more extreme positive and negative values of $S$. DNAm sites with extreme negative estimates of $S(S<-1)$ are annotated to a variety of genes including those involved in transcription $(A T F 7 I P)^{36}$ and tumour suppression $(S C R / B)^{37}$. DNAm sites with extreme positive estimates of $S(S>1)$ are annotated to a variety of genes including cg07175007 ( $\mathrm{S}=1.13, \mathrm{SD}=0.56)$ 
near UHMK1 associated with the cell cycle ${ }^{38}$ and $\operatorname{cg} 0479814(S=0.65, S D=0.60)$ near $S M Y D 3$ a histone methyltransferase ${ }^{39}$ (Table S1).

As a sensitivity analysis we implemented models to account for genetic drift $^{7}$ (Figure S4), which suggest that drift may be important but is not the sole driver of the signal of selection, supporting the hypothesis that this captures real biological processes. However, we cannot rule out that any specific effect was not caused by genetic drift.

\section{Polygenicity is associated with selection}

BayesNS estimates polygenicity as the proportion of $200 \mathrm{~kb}$ genomic 'windows' with non-zero effects' In contrast to findings from GoDMC ${ }^{19}$, our results suggest that the genetic architecture of DNAm is not very polygenic (highly heritable DNAm sites: $\bar{\pi}=0.04 \%$, ESS DNAm sites $\bar{\pi}=0.04 \%$, PhenoAge DNAm sites: $\bar{\pi}=0.06 \%$; Table 1 ). This finding is in part due to the bimodality of the effect size distribution in DNAm: we lack power to capture polygenic trans mQTLs with low effect sizes, whilst we are powered to detect large cis mQTL effects. However, it may also reflect the role of many DNAm sites in biological pathways, having a specific biological purpose but either affecting, or being affected by, many other processes.

We additionally investigated the number of SNPs (N SNPs) highly associated with each DNAm site (posterior inclusion probability; PIP >=0.8). Across all three sets of DNAm sites, we find a negative relationship between $S$ and N SNPs for DNAm sites (regression coefficient for highly heritable DNAm sites; -0.09; $p<2.2 \times 10^{-16}$, ESS DNAm sites; $-0.10 ; p<2.2 \times 10^{-16}$, PhenoAge DNAm sites; $-0.14 ; p=$ 0.00064; Figure 2). In addition, we find that SNPs associated with DNAm sites with negative estimates of $S$ have lower mean estimates of variance explained (VE) compared to those with positive estimates of $S$ (Figure 3). Polygenicity is therefore associated with selection, with DNAm associated with few mQTLs being the only class of positive selection, and highly polygenic DNAm being subject to strictly negative selection. Further, positively selected DNAm tends to have almost all of the heritability accounted for by identifiable $\mathrm{mQTLS}$.

\section{Relationship between selection estimates and traditional selection measures}

We additionally investigated whether estimates of $S$ correlate with five selection metrics: SDS ${ }^{17}, F_{s t}{ }^{40}$, integrated haplotype score (iHS) ${ }^{41}$, cross-population extended haplotype homozygosity XPEHH ${ }^{42}(\mathrm{CEU}$ v. YRI) and XPEHH (CEU v. CHB) in sets of 'high' PIP (PIP > 0.1) and 'all' PIP (PIP >=0.001) SNPs for each DNAm site. Values of $S$ have the highest correlation with $F_{s t}{ }^{40}(0.193$; Figure S5A, 0.113; Figure S5C, 0.151; Figure S5E, for highly heritable, ESS and PhenoAge DNAm sites respectively), however, when we include 'all' possible SNPs, even though we weight by $P I P$, the correlation becomes negative and tends to decrease in magnitude (-0.045; Figure S5B, -0.127; Figure S5D, -0.091; Figure S5F). This implies that cis or strongly acting trans SNPs are selected differently to the bulk DNA associations, i.e. that they are selected via a different mechanism, and that the low PIP SNPs are subject to a diversity of pathways, hence leading to an average selection close to 0 (Table 1). We additionally calculate 


\begin{tabular}{|c|c|c|c|c|c|c|c|c|c|}
\hline DNAm sites & $\overline{\boldsymbol{S}}$ & $\operatorname{SD}(\bar{S})$ & $\overline{h_{S N P}^{2}}(\%)$ & $\operatorname{SD}\left(\overline{h_{S N P}^{2}}\right)$ & $\bar{\pi}(\%)$ & $\mathrm{SD}(\bar{\pi})$ & $\overline{h_{2}}(\%)$ & $\mathrm{SD}\left(\overline{h_{2}}\right)$ & Convergence \\
\hline Highly heritable $(n=1804)$ & -0.15 & 0.46 & 30.2 & 12.4 & 0.04 & 0.02 & 89.9 & 5.9 & $90.2 \%(1804 / 2000)$ \\
\hline ESS $(n=887)$ & -0.14 & 0.50 & 26.6 & 13.0 & 0.04 & 0.02 & 78.8 & 20.4 & $56.1 \%(887 / 1580)$ \\
\hline PhenoAge $(n=74)$ & 0.04 & 0.48 & 14.8 & 9.7 & 0.06 & 0.04 & 53.4 & 23.0 & $14.4 \%(74 / 513)$ \\
\hline
\end{tabular}

SD; standard deviation, $h_{S N P}^{2}$; SNP based heritability and $\pi$, polygenicity, $h_{2}$; twin heritability estimates ${ }^{12}$ of 2000 heritable DNAm sites, 1580 ESS sites and 513 PhenoAge DNAm sites, Convergence; percentage of DNAm sites passing MCMC convergence checks

\section{Biological properties of DNAm sites under selection}

The magnitude of $S$ is related to the 'strength of selection on trait-associated SNPs'3. To understand whether DNAm traits under 'stronger' selection had biological relevance, we assessed whether DNAm sites with estimates of $S \leq-0.5$ and $\geq 0.5$ were enriched or depleted for predicted chromatin states ${ }^{44}$. The positive highly heritable DNAm sites $(n=212, S \geq 0.5$;) showed the strongest enrichment (qvalue $<0.05$ ) for enhancers (Odds Ratio; ORs EnhW1 1.75-3.21; ORs EnhW2 1.72-2.57) and promoters (PromP ORs=1.84-3.26; PromU ORs=1.52-2.18) (Figure 4A; Table S2). The negative highly heritable DNAm sites ( $n=376, S \leq-0.5$; Figure 4B; Table S2) showed only enrichment for poised promoters (PromP, OR=2.1) but not for transcription activity. Both positive ( $\mathrm{n}=123, S \geq 0.5)$ and negative ( $\mathrm{n}=218, S \leq-0.5$ ) ESS DNAm sites also show enrichment for poised promoters (PromP) across all tissue types (positive ORs 2.24-5.1 negative ORs: 1.79-3.64; Figure S7; Tables S3-S4). Poised chromatin is associated with both activating and repressing histone modifications and has been proposed to play a role in the prevention of DNAm ${ }^{45}$. DNAm sites showing signatures of selection are therefore enriched for bivalent chromatin structure associated with silencing genes whilst keeping them ready for activation ${ }^{46}$. CpG rich promoters have been shown to be subject to 'epigenetic buffering' against the effects of random mutations due to their association with housekeeping genes ${ }^{20}$.

We additionally assessed enrichment of 167 transcription factor binding sites (TFBSs) in 127 different cell types comprising 30 tissues ${ }^{47}$. Transcription factors have previously been shown to be under weak purifying selection, with a limited minority exhibiting signatures of positive selection ${ }^{48}$. Arbiza et al., find evidence of positive selection on GATA- binding zinc finger proteins ${ }^{48}$. Though, we do not see evidence of enrichment for TFBS for our DNAm sites of interest (Tables S6-S9). 


\section{BMI and birthweight associated DNAm sites show signatures of selection}

We used BayesNS to estimate $S, h_{S N P}^{2}$ and $\pi$ for 893 DNAm sites associated with birthweight ${ }^{31}$ and 243 DNAm sites associated with $\mathrm{BMI}^{30}$ in EWAS of individuals of European ancestry ${ }^{49} .220$ birthweightassociated DNAm sites (24.6\%) and 42 BMI-associated DNAm sites (17.0\%) passed MCMC convergence tests (Figure S3). As with the highly heritable, ESS and PhenoAge DNAm sites, MAFdependent genetic architectures estimated from genotype and DNAm revealed the action of both positive and negative selection for BMI $(\bar{S} ; 0.04 ;$ SD; 0.59 , range: $-1.09: 0.98)$ and birthweight $(\bar{S} ;-0.05$, SD; 0.59, range: $-1.81: 0.98$ ) associated DNAm sites (Figure 6; Table S10). Birthweight-associated cg16875057 has an $S$ estimate of -1.81 and is annotated to the STK39 gene which is associated with the cellular stress response pathway and hypertension ${ }^{50}$. In addition, birthweight-associated cg07157107 $(S=0.98)$ is associated with the nicotinic receptor CHRNA6, positive selection has previously been reported on genomic regions containing nicotinic receptor genes ${ }^{51}$. In contrast, a previous study using BMI and birthweight GWA loci found only evidence of negative selection ${ }^{3}$. After adjustment for non-random properties of the DNAm sites, we found that birthweight associated DNAm sites showed an enrichment of negative estimates of $S$ as compared to heritability matched background DNAm sites (Table 2). To assess whether biological pathways were enriched among the DNAm sites with extreme $S$ we performed GOterm enrichment analysis, however none of the pathways showed evidence of enrichment.

Table 2| Birthweight-associated DNAm sites are enriched for negative estimates of $S$

\begin{tabular}{lcccc}
\hline DNAm sites & Fisher's exact test $\boldsymbol{P}$-value & Odds ratio (OR) & Lower 95\% Cl & Upper 95\% Cl \\
\hline PhenoAge $(n=74)$ & 1 & 1.09 & 0.44 & 2.70 \\
\hline BMl-associated $(n=42)$ & 1 & 1.13 & 0.37 & 3.45 \\
\hline Birthweight $(n=220)$ & $3.432 \times 10^{-6}$ & 0.29 & 0.16 & 0.50 \\
\hline
\end{tabular}




\section{Discussion}

In this study, we have characterized the genetic architecture of DNA methylation at individual DNAm sites measured on the $450 \mathrm{k}$ array ${ }^{29}$. Specifically, we consider estimates of polygenicity, SNP-based heritability and the joint-distribution of effect size and MAF for 1804 highly heritable DNAm sites, 887 ESS DNAm sites and 74 PhenoAge DNAm sites. Unlike previous work looking at complex traits and gene expression which find evidence of negative selection exclusively ${ }^{3,5,6}$, across all sets of DNAm sites we find evidence of both positive $(S>0)$ and negative selection $(S<0)$. These findings support previous research showing an enrichment of MQTLs among SNPs with signatures of positive selection, plus a negative relationship between MAF and mQTL effect size ${ }^{19}$. We were able to estimate $S$ at individual DNAm sites allowing us to identify specific DNAm sites with extreme estimates of $S$. In addition, we considered DNAm sites associated with complex traits that have previously been shown to exhibit signatures of negative selection with Bayes $S^{3}$. DNAm sites associated with birthweight in EWAS had a higher proportion of DNAm sites with negative estimates of $S$ compared to heritability matched DNAm sites.

For traits which are less polygenic it can be particularly hard to separate the actions of natural selection and genetic drift, which can generate extreme changes to the frequency of SNPs between human populations such as our study population (Europeans) and the common ancestor in which DNAm evolved (predating the out-of-Africa event) ${ }^{7}$. A Bayesian model accounting for genetic drift found that individual estimates of $S$ could be due to either selection or genetic drift, but collectively DNAm was impacted by both positive and negative selection, not explainable by genetic drift alone ${ }^{7}$.

In addition to this, we were also able to characterize SNP based heritability and polygenicity for individual DNAm sites. Across all DNAm sites, average SNP based heritability was $28.6 \%$. This is higher than previous estimates looking at ARIES data, but likely reflects the fact we are constrained to considering DNAm sites which pass convergence checks ${ }^{52}$. For each group of DNAm sites, average polygenicity was low. We found a striking relationship between polygenicity and selection, with positively selected DNAm associated with only a small number of mQTLs which together explained most of the heritability of the trait. Conversely, negatively selected DNAm is likely to be explained by more mQTLs, many of which we lack statistical power to identify. The genetic architecture of DNAm has been shown to have a large cis-mQTL effect plus polygenic trans-mQTLs of low effect sizes ${ }^{19,52}$. Studies in larger and more diverse populations should be undertaken to further investigate the relationship between polygenicity and selection. Our results provide insight into how genetic architecture of individual DNAm sites has been influenced by natural selection.

There are several limitations of this study. The model is restricted to looking at DNAm sites which pass MCMC convergence checks, which typically are those with high heritability in twin studies ${ }^{12}$. In addition, we compared BayesNS estimates to other selection metrics ( $F_{s t}$, SDS, XPEHH, iHS), which are specialised to detect signatures of positive selection and have an estimate per SNP. This means that they do not make an ideal comparison group, since BayesS can be used to make inferences about both 
positive and negative selection and estimates are provided at the trait level. Blood cell counts have previously been reported to show signatures of negative selection ${ }^{6}$. Whilst our DNAm data has been adjusted for recorded cell counts ${ }^{19}$, the relationship between DNAm and blood cell counts ${ }^{53}$ could warrant further investigation in regards to whether it influences estimates of $S$. As mentioned, larger sample sizes are needed to detect $\mathrm{mQTLs}$ with low effect size which we are not powered to detect. Our study was also limited to the $450 \mathrm{k}$ array which measures $1.5 \%$ of the genome and is biased to promoters $^{29}$. Large epidemiological studies profiled with EPIC arrays ${ }^{54}$ (measuring regulatory elements) are expected to find additional signatures for selection.

Overall, our study finds evidence for both positive and negative selection in the genetic architecture of DNAm. We are unable to cleanly place DNAm in the causal pathway between genetic variation and selection. Our results are consistent with two competing hypotheses; firstly, that selection occurs on DNAm due to a biological function it has, or secondly DNAm is influenced by complex traits that are themselves the target of selection. The presence of both positive and negative selection is an indication that both pathways may play a role. Specifically, we hypothesise that DNAm may perform a biological function which is of less selective importance than the complex traits that have widespread impact on genome wide DNAm, swamping and confusing the signal with a mixture of proximal and distal signals. Future work looking into the biological relevance of individual DNAm sites with positive and negative estimates of $S$ could help to identify biological pathways which effect fitness. DNAm data from diverse individuals will be essential in separating the effects of drift and selection. Understanding the selective forces shaping DNAm could ultimately help identify potential targets for disease intervention. 


\section{Methods}

\section{Study Population}

Participants were from the Avon Longitudinal Study of Parents and Children (ALSPAC) ${ }^{55,56}$, a large prospective cohort study that recruited 14,541 pregnancies, resident in the Bristol and Avon area with expected delivery dates between the $1^{\text {st }}$ of April 1991 and the $31^{\text {st }}$ of December 1992. Full details of the cohort have been published elsewhere ${ }^{55,56}$. The study website contains details of all the data that are available through a fully searchable data dictionary (http://www.bristol.ac.uk/alspac/researchers/ourdata/). Written and informed consent has been obtained for all ALSPAC participants. Ethical approval for the study was obtained from the ALSPAC Ethics and Law Committee and the Local Research Ethics Committees (http://www.bristol.ac.uk/alspac/researchers/research-ethics/).

\section{ALSPAC genotype data}

ALSPAC mothers were genotyped using the Illumina Human660W-quad array at Centre National de Génotypage (CNG). ALSPAC offspring were genotyped using the Illumina HumanHap550 quad chip genotyping platforms by 23andMe subcontracting the Wellcome Trust Sanger Institute, Cambridge, UK and the Laboratory Corporation of America, Burlington, NC, USA. For ALSPAC mothers, SNPs with a MAF of $<1 \%$, a call rate of $<95 \%$, or evidence for violations of Hardy-Weinberg equilibrium $(p<1 \times$ $10^{-6}$ ) were removed. For ALSPAC offspring, SNPs with MAF of $<1 \%$, a call rate of $<95 \%$ or evidence for violations of Hardy-Weinberg equilibrium $\left(p<5 \times 10^{-7}\right)$, were removed. Cryptic relatedness within mothers and within offspring was measured as proportion of identity by descent (IBD < 0.1$)$. All individuals with non-European ancestry were removed. Imputation of ALSPAC genetic data was performed on a combined mother and child dataset using Impute2 against the 1000 Genomes Project Phase 1 Version 3 reference panel.

Linkage disequilibrium (LD) pruning was undertaken using PLINK ${ }^{57}$ using the following settings $\left(r^{2}=0.1\right.$, window size $=50 \mathrm{~kb}$ ). SNPs residing within the Major Histocompatibility Complex (MHC) (chr6: 25Mb: $35 \mathrm{Mb}$ ) and lactase (LCT) regions (chr2: $129 \mathrm{Mb}: 144 \mathrm{Mb}$ ) were removed as they are known to be under high selective pressure (build 37). This left 474,939 SNPs available for analysis.

\section{DNA methylation data}

In ALSPAC, blood from 1018 mother-child pairs were selected for analysis as part of ARIES ${ }^{34}$ (http://www.ariesepigenomics.org.uk/). Following DNA extraction, samples were bisulphite converted using the Zymo EZ DNA Methylation ${ }^{\mathrm{TM}}$ kit (Zymo, Irvine, CA, USA), and DNA methylation was measured using the Illumina Infinium HumanMethylation450 (HM450) BeadChip. ARIES consists of DNAm measures at five time points (three time points for children: birth, childhood, and adolescence; and two for mothers: during pregnancy and at middle age). We utilised data on a total of 1774 individuals from the adolescence and middle age time points with both DNAm and genotype data (http://www.ariesepigenomics.org.uk/). 
DNAm was adjusted using the GoDMC pipeline (described elsewhere) ${ }^{19}$. Briefly, we adjusted for sex, age at measurement, batch variables, smoking and predicted cell counts. Genetic principal components (PCs), non-genetic DNAm PCs were also calculated using the GoDMC pipeline, and a genetic kinship matrix was fitted using GRAMMAR ${ }^{58}$. The residuals of these analyses were rank transformed to have a mean 0 and variance 1 .

We selected the following DNAm sites for analyses:

1. 2000 DNAm sites with twin heritability estimates between 0.99 and $0.79^{12}$ (referred to as 'highly heritable' DNAm sites)

2. 1580 DNAm sites identified as having greater epigenetic similarity than can be explained genetically, so-called 'epigenetic supersimilarity' (ESS) DNAm sites ${ }^{14}$

3. 513 DNAm sites forming an epigenetic biomarker of aging, PhenoAge ${ }^{32}$, that is predictive of all-cause mortality

4. 243 DNAm sites associated with BMI $\left(p<1 \times 10^{-4}\right)$ in a discovery EWAS of 2707 individuals of European ancestry ${ }^{30}$. Results were obtained and downloaded from the EWAS catalog ${ }^{49}$

5. 893 DNAm sites associated with birthweight $\left(p<1 \times 10^{-4}\right)$ in a cord blood EWAS meta-analysis of 6023 individuals of European ancestry ${ }^{31}$. Results were obtained and downloaded from the EWAS catalog ${ }^{49}$

To serve as comparison groups, we additionally ran BayesNS on 513, 243 and 893 background DNAm sites matched on $\mathrm{GC} / \mathrm{CpG}$ content and heritability to PhenoAge, BMI and birthweight associated sites respectively.

\section{BayesNS Analysis}

BayesS is a Bayesian mixed linear model (MLM) method that can jointly estimate SNP-based heritability $\left(h_{S N P}^{2}\right)$, polygenicity $(\pi)$ and the joint distribution between MAF and SNP effect size $(S)^{3}$. The relationship between MAF and effect size is used to make inferences about natural selection and is modelled using the following mixture distribution as a prior for each SNP effect:

$$
\beta_{j} \sim N\left(0,\left[2 p_{j}\left(1-p_{j}\right)\right]^{S} \sigma_{\beta}^{2}\right) \pi+\varphi(1-\pi)
$$

Where $\beta_{j}$ is the effect of a SNP $j, p_{j}$ is the MAF, $\sigma_{\beta}^{2}$ is the variance of SNP effects under a neutral model, $\varphi$ is a point mass at zero and $\pi$ is polygenicity (defined as the proportion of SNPs with non-zero effects). $S$ is the estimated selection coefficient, when $S>0$ effect size is positively related to MAF and when $S<0$ effect size is negatively related to MAF. When $S=0$, effect size and MAF are unrelated. Bayes $S$ uses a Markov Chain Monte Carlo (MCMC) algorithm for posterior inference. SNP-based heritability is estimated using the sampled effects of SNPs in the MCMC. We applied a nested version of BayesS (BayesNS) recommended for traits with low polygenicity such as DNAm. BayesNS considers SNPs together in non-overlapping windows and skips over regions of zero effect. SNPs in the same window 
are individually modelled as in BayesS, but also collectively considered as a window effect. The length of each window was set as $200 \mathrm{~kb}$, replicating the window size selected for analyses of gene expression ${ }^{3}$. Polygenicity $(\pi)$ here is considered as the proportion of windows with nonzero effects. We considered each DNAm site as an individual trait in our analyses.

For the MCMC algorithm we set the chain length to 30,000 iterations with the first 10,000 discarded as burn-in. We plotted MCMC trace plots using bayesplot (http://mc-stan.org/bayesplot/) to visually assess convergence of the MCMC algorithm. In addition, we ran the Raftery and Lewis ${ }^{35}$ run length control diagnostic in coda and selected a threshold of less than 10 for the dependence factor (I) (Figures $\mathrm{S} 2 ; \mathrm{S} 3)$. MCMC convergence checks were performed in $\mathrm{R}$ version 3.6.2.

\section{Accounting for Genetic Drift}

We additionally ran a Bayesian model for genetic architecture which accounts for genetic drift. We used the MCMC algorithm from Ashraf and Lawson $(2021)^{7}$ and applied it to the highly heritable, ESS and PhenoAge DNAm sites. Specifically, the prior for the selection coefficient $S \sim U(-2,2)$, and for the standard deviation of $\beta$ is $\sigma_{\beta} \sim U(0,2)$. Unlike in BayesS where $\beta_{\mathrm{I}}$ is a prior, it and the allele frequency $p_{\mathrm{I}}$ are treated as data, via the same relationship:

$$
\beta_{i} \sim \operatorname{Normal}\left(0, \sigma_{\beta}^{2}\left[p_{i}\left(1-p_{i}\right)\right]^{S}\right)
$$

The drift model below is the appropriate model accounting for genetic drift. To furter check that our results are consistent with BayesS we report results for three models:

1. Null model: this extends the likelihood to account for PIP. $p_{\mathrm{i}}$ is considered fixed, and the likelihood from each SNP weighted by its inclusion probability $w_{i}=P I P(i)$. The log-likelihood is $L=\sum_{i=1}^{N} w_{i} \log p\left(\beta_{i} ; 0, \sigma_{\beta}^{2}\left[p_{i}\left(1-p_{i}\right)\right]^{S}\right)$, where $p$ is the Normal distribution density.

2. No-drift model: no genetic drift but accounting for PIP and uncertainty in $\beta_{\mathrm{i}}, p_{\mathrm{i}}$ is considered fixed, the observed effect size $\beta_{i} \sim N\left(b_{i}, \sigma_{b}^{2}\right)$ where $\sigma_{b}$ is the standard error of the estimate. Then following above $L=\sum_{i=1}^{N} w_{i} \log p\left(b_{i} ; 0, \sigma_{\beta}^{2}\left[p_{i}\left(1-p_{i}\right)\right]^{S}\right)$.

3. Drift model: accounting for genetic drift, PIP and uncertainty in $\beta_{\mathrm{i}}$. Drift is modelled with the Baldings-Nichols model. Let $f_{i}$ be the true frequency in the ancestral population and $p_{i}$ be observed as above. Then $p_{i} \sim \operatorname{Beta}\left(f_{i}\left(1-F_{s t}\right) / F_{s t},\left(1-f_{i}\right)\left(1-F_{s t}\right) / F_{s t}\right), \beta_{i} \sim N\left(b_{i}, \sigma_{b}^{2}\right)$ and $L=$ $\sum_{i=1}^{N} w_{i} \log p\left(b_{i} ; 0, \sigma_{\beta}^{2}\left[f_{i}\left(1-f_{i}\right)\right]^{S}\right)$.

$F_{s t}$ is set to 0.15 , matching the empirical estimate from the out-of-Africa bottleneck at these SNPs as in the original implementation? 


\section{Calculating variance explained}

Following BayesNS analyses we investigated SNPs associated with DNAm at individual DNAm sites. We selected SNPs with a high posterior inclusion probability $(P I P)>=0.8$. We calculated the number of SNPs (nSNPs) with a PIP >= 0.8 for each DNAm site.

For each DNAm site we calculated variance explained for SNPs with PIP >=0.8:

$$
\beta_{j}^{2} 2 p_{j}\left(1-p_{j}\right)
$$

Where $\beta_{j}$ is the effect of a SNP $j, p_{j}$ is the MAF.

\section{Analysing LD score and selection metrics}

To determine whether our results were influenced by LD, we additionally looked up European LD scores from the 1000 Genomes Project for each of these SNPs ${ }^{43}$. To compare BayesNS estimates of $S$ with other selection scores we used metrics from the 1000 Genomes Selection Browser 1.0. We selected the same five annotations used in GoDMC ${ }^{19}$, reflecting selection over different timescales: singleton density score (SDS ${ }^{17}$; UK10K), $F_{s t}{ }^{40}$ (Global $F_{s t}$ (CEU vs. YRI vs. CHB)), integrated haplotype score (iHS;CEU) ${ }^{41}$, cross population extended haplotype homozygosity (XPEHH; CEU vs. YRI) and XPEHH $(\mathrm{CEU} \text { vs. } \mathrm{CHB})^{42}$. These methods focus on positive selection ${ }^{18} ; F_{s t}$ is based on population differentiation ${ }^{40}, \mathrm{XPEHH}$ is a cross-population test based on extended haplotype homozygosity $(\mathrm{EHH})$, $\mathrm{iHS}$ is defined as the log ratio of integrated haplotype scores for each allele in a single population ${ }^{41}$. SDS measures very recent changes in allele frequency from contemporary genome sequences and has been applied to the UK10K dataset ${ }^{17}$.

For each DNAm site we calculated the mean value for each of these selection scores for SNPs with $P I P>0.1$ and $P I P>0.001$ respectively. Each mean was weighted by the SNPs PIP value. We used ggpairs in R version 3.6.2 to plot pairwise distributions of BayesNS, LDSC and selection scores and to compute the Pearson correlation coefficient between these variables.

\section{Enrichment Analysis}

We assessed enrichment or depletion of DNAm sites for 25 chromatin states and TFBSs in 127 different cell types comprising 30 tissues. These data were generated by the Roadmap Epigenomics Project ${ }^{44}$ (http://www.roadmapepigenomics.org/) and ENCODE (https://www.encodeproject.org/). We used Locus Overlap Analysis (LOLA) ${ }^{59}$ (Bioconductor version: Release 3.12) to perform a two-sided Fisher's exact test. Since the magnitude of $S$ reflects the strength of selection we selected DNAm sites with estimates of $S \geq 0.5$ and $S \leq-0.5$ for analyses. Background sites from the HumanMethylation450 array were matched on GC and CpG content and heritability prior to analysis (Figure S6), as differential GC content/heritability's between the sites of interest and background sites may bias the results. Analyses were conducted using R v. 3.6.2. 
Four groups of DNAm sites were considered for enrichment analysis:

1. 212 highly heritable DNAm sites with estimates of $S \geq 0.5$ (positive highly heritable DNAm sites)

2. 376 highly heritable DNAm sites with estimates of $S \leq-0.5$ (negative highly heritable DNAm sites)

3. 123 ESS DNAm sites with estimates of $S \geq 0.5$ (positive ESS DNAm sites)

4. 218 ESS DNAm sites with estimates of $S \leq-0.5$ (negative ESS DNAm sites)

\section{DNAm and complex traits}

We ran BayesNS on DNAm sites associated with BMI and birthweight in two large-scale EWAS of participants with European ancestry. In addition, we ran BayesNS on DNAm sites not associated with the traits of interest, matched on GC/CpG content and heritability. We split DNAm sites in each set (PhenoAge, BMI-associated DNAm sites, birthweight-associated DNAm sites and matched background DNAm sites) into two groups: DNAm sites with negative estimates of $S \leq-0.5$ and DNAm sites with estimates of $S>-0.5$. We then performed one-sided Fisher's exact tests to investigate whether DNAm sites associated with PhenoAge, BMI and birthweight exhibit statistically different estimates of $S$ compared to a set of matched background DNAm sites. We additionally performed GOterm enrichment analysis implemented in missmethyl|601. 


\section{Acknowledgements}

We are extremely grateful to all the families who took part in this study, the midwives for their help in recruiting them, and the whole ALSPAC team, which includes interviewers, computer and laboratory technicians, clerical workers, research scientists, volunteers, managers, receptionists, and nurses.

\section{Funding}

The UK Medical Research Council and Wellcome (Grant ref: 217065/Z/19/Z) and the University of Bristol provide core support for ALSPAC. This publication is the work of the authors who will serve as guarantors for the contents of this paper. A comprehensive list of grants funding is available on the ALSPAC website (http://www.bristol.ac.uk/alspac/external/documents/grant-acknowledgements.pdf). GWAS data was generated by Sample Logistics and Genotyping Facilities at Wellcome Sanger Institute and LabCorp (Laboratory Corporation of America) using support from 23andMe. This study and C.H. were supported by a 4-year studentship fund from the Wellcome Trust Molecular, Genetic and Lifecourse Epidemiology Ph.D. programme at the University of Bristol (108902/B/15/Z). J.L.M, D.J.L., T.R.G., S.R are members of the UK Medical Research Council Integrative Epidemiology Unit at the University of Bristol (MC_UU_00011/4, MC_UU_00011/5). For the purpose of Open Access, the author has applied a CC BY public copyright licence to any Author Accepted Manuscript version arising from this submission.

\section{Author contributions}

Analysed the data: C.H., G.H., J.L.M., D.J.L., T.R.G., S.R.

Contributed data: GoDMC, ALSPAC

Designed and managed the study: J.L.M., D.J.L., T.R.G., S.R.

Wrote the manuscript: C.H., J.L.M., D.J.L., T.R.G., S.R. 


\section{References}

1. Edwards, S. L., Beesley, J., French, J. D. \& Dunning, M. Beyond GWASs: Illuminating the dark road from association to function. Am. J. Hum. Genet. 93, 779-797 (2013).

2. Pritchard, J. K., Pickrell, J. K. \& Coop, G. The Genetics of Human Adaptation: Hard Sweeps, Soft Sweeps, and Polygenic Adaptation. Curr. Biol. 20, R208-R215 (2010).

3. Zeng, J. et al. Signatures of negative selection in the genetic architecture of human complex traits. Nat. Genet. 50, 746-753 (2018).

4. O'Connor, L. J. et al. Extreme Polygenicity of Complex Traits Is Explained by Negative Selection. Am. J. Hum. Genet. 105, 456-476 (2019).

5. Speed, D., Holmes, J. \& Balding, D. J. Evaluating and improving heritability models using summary statistics. Nat. Genet. 52, 458-462 (2020).

6. Schoech, A. P. et al. Quantification of frequency-dependent genetic architectures in 25 UK Biobank traits reveals action of negative selection. Nat. Commun. 10, 790 (2019).

7. Ashraf, B. \& Lawson, D. J. Genetic drift from the out-of-Africa bottleneck leads to biased estimation of genetic architecture and selection. Eur. J. Hum. Genet. 2020.08.17.254110 (2021) doi:10.1038/s41431-021-00873-2.

8. Giambartolomei, C. et al. Bayesian Test for Colocalisation between Pairs of Genetic Association Studies Using Summary Statistics. PLoS Genet. 10, e1004383 (2014).

9. Giambartolomei, C. et al. A Bayesian framework for multiple trait colocalization from summary association statistics. Bioinformatics 34, 2538-2545 (2018).

10. Horvath, S. DNA methylation age of human tissues and cell types. Genome Biol. 14, R115 (2013).

11. Joehanes, R. et al. Epigenetic Signatures of Cigarette Smoking. Circ. Cardiovasc. Genet. 9, 436-447 (2016).

12. Van Dongen, J. et al. Genetic and environmental influences interact with age and sex in shaping the human methylome. Nat. Commun. 7, 11115 (2016).

13. Fraga, M. F. et al. Epigenetic differences arise during the lifetime of monozygotic twins. Proc. Natl. Acad. Sci. U. S. A. 102, 10604-10609 (2005).

14. van Baak, T. E. et al. Epigenetic supersimilarity of monozygotic twin pairs. Genome Biol. 19, 2 (2018).

15. Ecker, S., Pancaldi, V., Valencia, A., Beck, S. \& Paul, D. S. Epigenetic and Transcriptional Variability Shape Phenotypic Plasticity. Bioessays 40, (2018).

16. Min, J. L. et al. Genomic and phenotypic insights from an atlas of genetic effects on DNA methylation. Nat. Genet. 53, 1311-1321 (2021).

17. Field, Y. et al. Detection of human adaptation during the past 2000 years. Science (80-. ). 354, 760-764 (2016).

18. Pybus, M. et al. 1000 Genomes Selection Browser 1.0: A genome browser dedicated to signatures of natural selection in modern humans. Nucleic Acids Res. 42, D903-D909 (2014).

19. Min, J. L. et al. Genomic and phenomic insights from an atlas of genetic effects on DNA methylation. medRxiv 2020.09.01.20180406 (2020) doi:10.1101/2020.09.01.20180406. 
20. Onuchic, V. et al. Allele-specific epigenome maps reveal sequence-dependent stochastic switching at regulatory loci. Science 361, (2018).

21. Fagny, M. et al. The epigenomic landscape of African rainforest hunter-gatherers and farmers. Nat. Commun. 6, 10047 (2015).

22. Lonsdale, J. et al. The Genotype-Tissue Expression (GTEx) project. Nat. Genet. 45, 580-585 (2013).

23. Glassberg, E. C., Gao, Z., Harpak, A., Lan, X. \& Pritchard, J. K. Evidence for weak selective constraint on human gene expression. Genetics 211, 757-772 (2019).

24. Korthauer, K. \& Irizarry, R. Genome-wide repressive capacity of promoter DNA methylation is revealed through epigenomic manipulation. bioRxiv 381145 (2018) doi:10.1101/381145.

25. Ford, E. et al. Frequent lack of repressive capacity of promoter DNA methylation identified through genome-wide epigenomic manipulation. bioRxiv 170506 (2017) doi:10.1101/170506.

26. Levenson, V. V. DNA methylation as a universal biomarker. Expert Rev. Mol. Diagn. 10, 481488 (2010).

27. Petronis, A. Epigenetics as a unifying principle in the aetiology of complex traits and diseases. Nature 465, 721-727 (2010).

28. Héberlé, É. \& Bardet, A. F. Sensitivity of transcription factors to DNA methylation. Essays Biochem. 63, 727-741 (2019).

29. Bibikova, M. et al. High density DNA methylation array with single $\mathrm{CpG}$ site resolution. Genomics 98, 288-295 (2011).

30. Wahl, S. et al. Epigenome-wide association study of body mass index, and the adverse outcomes of adiposity. Nature 541, 81-86 (2017).

31. Küpers, L. K. et al. Meta-analysis of epigenome-wide association studies in neonates reveals widespread differential DNA methylation associated with birthweight. Nat. Commun. 10, 1893 (2019).

32. Levine, M. E. et al. An epigenetic biomarker of aging for lifespan and healthspan. Aging (Albany. NY). 10, 573-591 (2018).

33. Williams, G. C. Pleiotropy, Natural Selection, and the Evolution of Senescence. Evolution (N. Y). 11, 398-411 (1957).

34. Relton, C. L. et al. Data resource profile: Accessible resource for integrated epigenomic studies (ARIES). Int. J. Epidemiol. 44, 1181-1190 (2015).

35. Raftery, A. E. \& Lewis, S. M. [Practical Markov Chain Monte Carlo]: Comment: One Long Run with Diagnostics: Implementation Strategies for Markov Chain Monte Carlo. Stat. Sci. 7, 493497 (1992).

36. Liu, L. et al. MCAF1/AM is involved in Sp1-mediated maintenance of cancer-associated telomerase activity. J. Biol. Chem. 284, 5165-5174 (2009).

37. Zhang, W. et al. A global transcriptional network connecting noncoding mutations to changes in tumor gene expression. Nat. Genet. 50, 613-620 (2018).

38. Barbutti, I. et al. The U2AF homology motif kinase 1 (UHMK1) is upregulated upon hematopoietic cell differentiation. Biochim. Biophys. Acta - Mol. Basis Dis. 1864, 959-966 (2018). 
39. Sun, J., Li, Z. \& Yang, N. Mechanism of the Conformational Change of the Protein Methyltransferase SMYD3: A Molecular Dynamics Simulation Study. Int. J. Mol. Sci. 22, 7185 (2021).

40. Weir, B. S. \& Cockerham, C. C. Estimating F-statistics for the analysis of population structure. Evolution (N. Y). 38, 1358-1370 (1984).

41. Voight, B. F., Kudaravalli, S., Wen, X. \& Pritchard, J. K. A map of recent positive selection in the human genome. PLoS Biol. 4, 0446-0458 (2006).

42. Sabeti, P. C. et al. Genome-wide detection and characterization of positive selection in human populations. Nature 449, 913-918 (2007).

43. Bulik-Sullivan, B. et al. LD score regression distinguishes confounding from polygenicity in genome-wide association studies. Nat. Genet. 47, 291-295 (2015).

44. Roadmap Epigenomics Consortium et al. Integrative analysis of 111 reference human epigenomes. Nature 518, 317-329 (2015).

45. Lesch, B. J. \& Page, D. C. Poised chromatin in the mammalian germ line. Development 141, 3619-3626 (2014).

46. Hoffman, M. M. et al. Integrative annotation of chromatin elements from ENCODE data. Nucleic Acids Res. 41, 827-841 (2013).

47. The, E. P. C. et al. An integrated encyclopedia of DNA elements in the human genome. Nature 489, 57 (2012).

48. Arbiza, L. et al. Genome-wide inference of natural selection on human transcription factor binding sites. Nat. Genet. 45, 723-729 (2013).

49. Battram, T. et al. The EWAS Catalog: a database of epigenome-wide association studies. OSF Prepr. 4 (2021) doi:10.31219/osf.io/837wn.

50. Wang, Y. et al. Whole-genome association study identifies \&lt;em\&gt;STK39\&lt;/em\&gt; as a hypertension susceptibility gene. Proc. Natl. Acad. Sci. 106, 226 LP - 231 (2009).

51. Sadler, B. et al. Positive Selection on Loci Associated with Drug and Alcohol Dependence. PLoS One 10, e0134393 (2015).

52. Gaunt, T. R. et al. Systematic identification of genetic influences on methylation across the human life course. Genome Biol. 17, 61 (2016).

53. Houseman, E. A. et al. DNA methylation arrays as surrogate measures of cell mixture distribution. BMC Bioinformatics 13, 16 (2012).

54. Salas, L. A. et al. An optimized library for reference-based deconvolution of whole-blood biospecimens assayed using the Illumina HumanMethylationEPIC BeadArray. Genome Biol. 19, 64 (2018).

55. Boyd, A. et al. Cohort profile: The 'Children of the 90s'-The index offspring of the avon longitudinal study of parents and children. Int. J. Epidemiol. 42, 111-127 (2013).

56. Fraser, A. et al. Cohort profile: The avon longitudinal study of parents and children: ALSPAC mothers cohort. Int. J. Epidemiol. 42, 97-110 (2013).

57. Purcell, S. et al. PLINK: A tool set for whole-genome association and population-based linkage analyses. Am. J. Hum. Genet. 81, 559-575 (2007).

58. Aulchenko, Y. S., De Koning, D. J. \& Haley, C. Genomewide rapid association using mixed 
bioRxiv preprint doi: https://doi.org/10.1101/2021.11.25.469994; this version posted November $25,2021$. The copyright holder for this

preprint (which was not certified by peer review) is the author/funder, who has granted bioRxiv a license to display the preprint in perpetuity. It is made available under aCC-BY 4.0 International license.

model and regression: A fast and simple method for genomewide pedigree-based quantitative trait loci association analysis. Genetics 177, 577-585 (2007).

59. Sheffield, N. C. \& Bock, C. LOLA: Enrichment analysis for genomic region sets and regulatory elements in R and Bioconductor. Bioinformatics 32, 587-589 (2016).

60. Young, A. I. Solving the missing heritability problem. PLOS Genet. 15, e1008222 (2019).

61. Phipson, B., Maksimovic, J. \& Oshlack, A. missMethyl: an R package for analyzing data from Illumina's HumanMethylation450 platform. Bioinformatics 32, 286-288 (2016). 
A

A Highly heritable DNAm sites

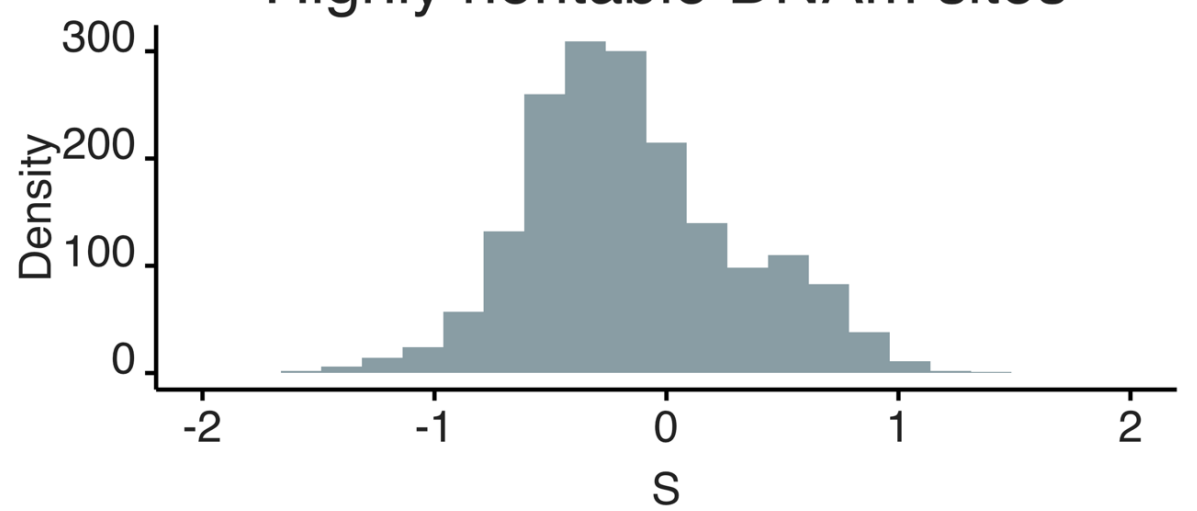

C

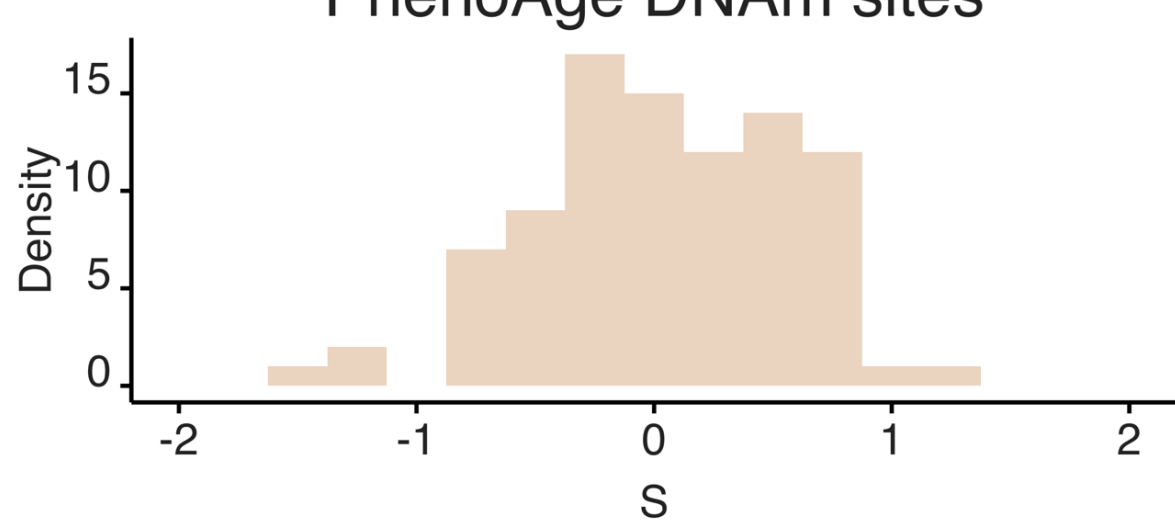

B

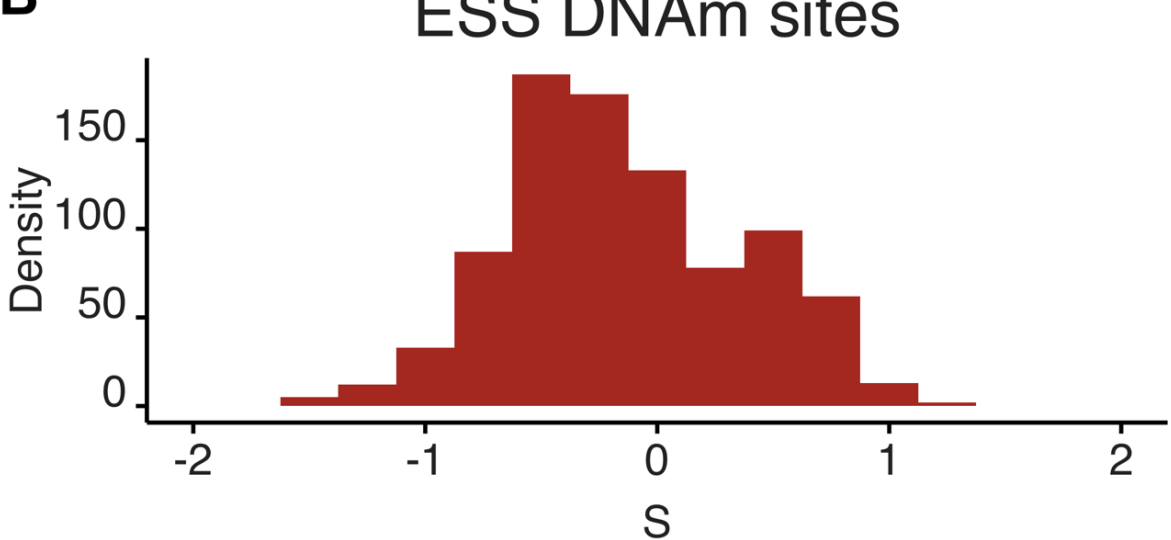

D

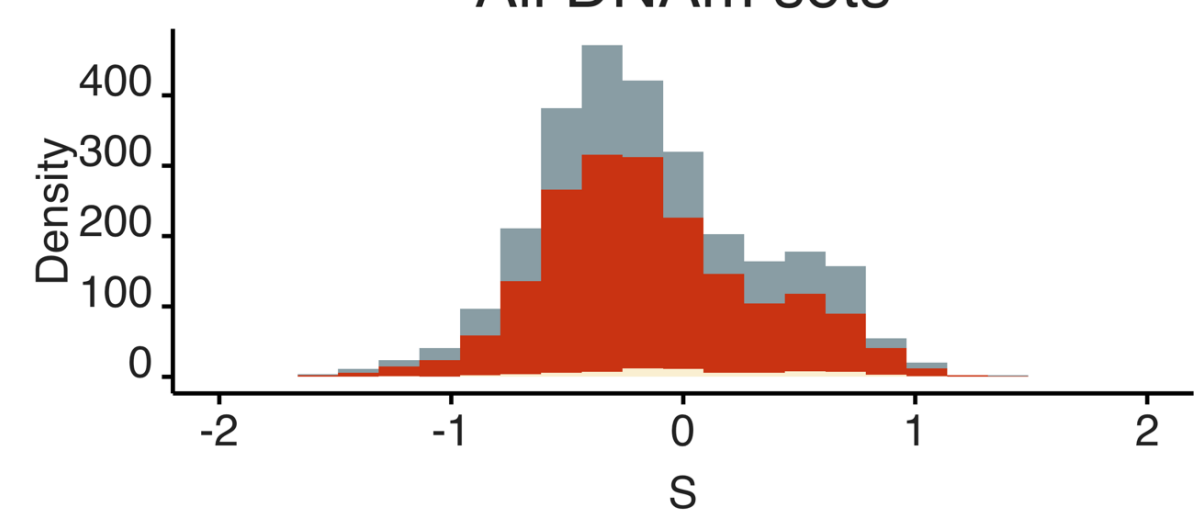

Figure 1| Estimates of $S$ from BayesNS. $S$ is estimated using the relationship between SNP effect size and MAF, when $S=0$ SNP effect size is independent of MAF (neutral), $S>0$ indicates positive selection, $S<0$ indicates negative selection. Results are for (A) 1804 highly heritable DNAm sites, (B) 887 ESS DNAm sites and (C) 74 PhenoAge DNAm sites which passed MCMC convergence checks. All DNAm sites shown in (D) with highly heritable DNAm sites in grey, ESS DNAm sites in red and PhenoAge DNAm sites in beige. 
A Heritable DNAm sites

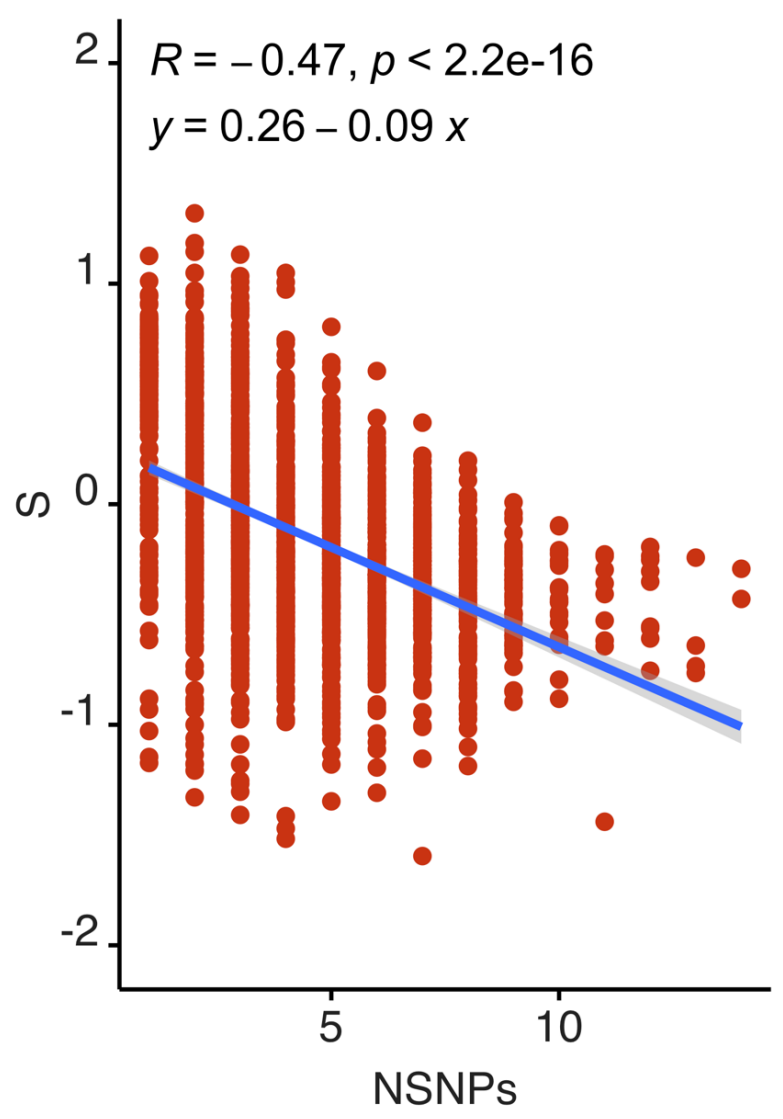

B

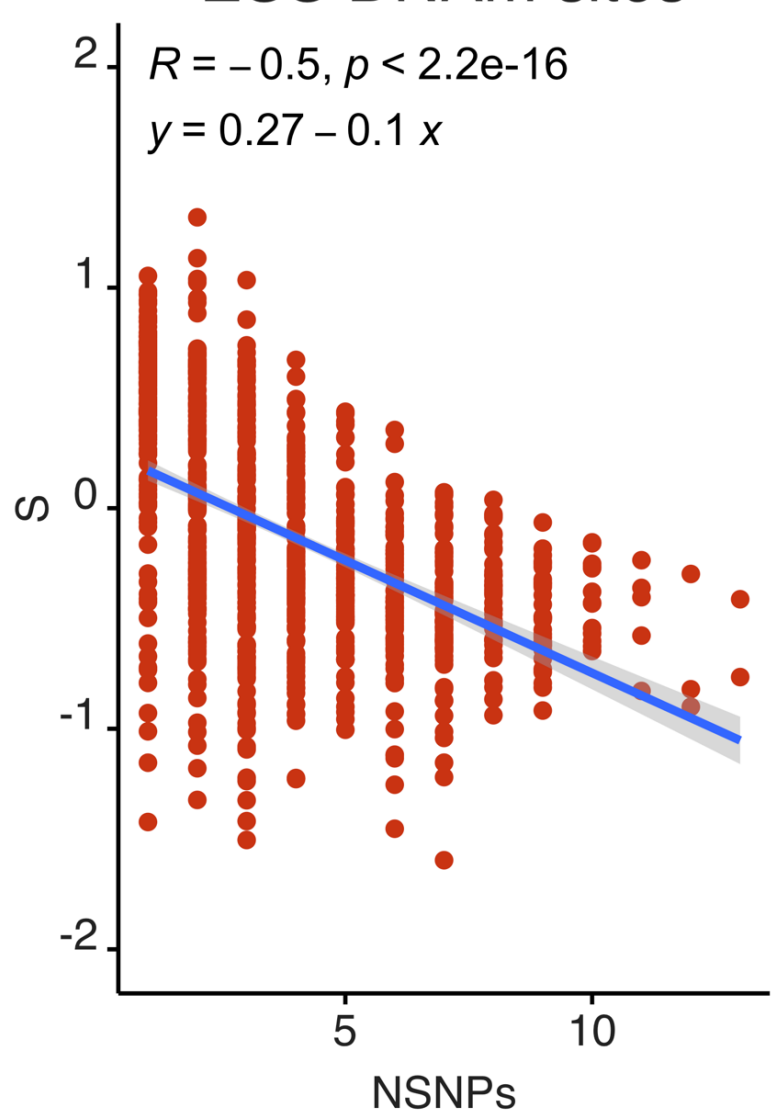

C PhenoAge DNAm sites

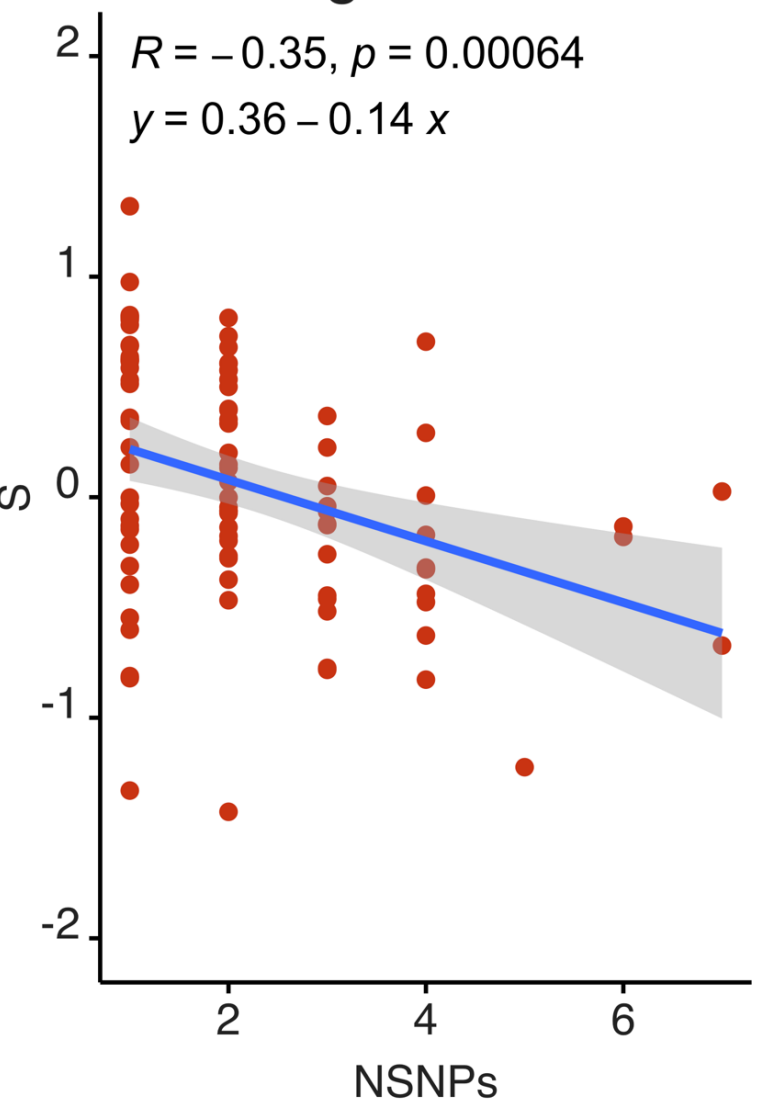

Figure 2 | Relationship between estimates of $S$ from BayesNS and NSNPs. For (A) 1804 highly heritable DNAm sites, (B) 887 ESS DNAm sites and (C) 74 PhenoAge DNAm sites. NSNPs calculated as the number of SNPs with a posterior inclusion probability (PIP) $>=0.8$ for each DNAm site and $S$ calculated from the relationship between SNP effect size and MAF. Slope for highly heritable DNAm sites $\left(-0.09 ; p<2.2 \times 10^{-16}\right)$, ESS DNAm sites $\left(-0.10 ; p<2.2 \times 10^{-16}\right)$, PhenoAge DNAm sites $(-0.14 ; p=0.00064)$. 
A Heritable DNAm sites

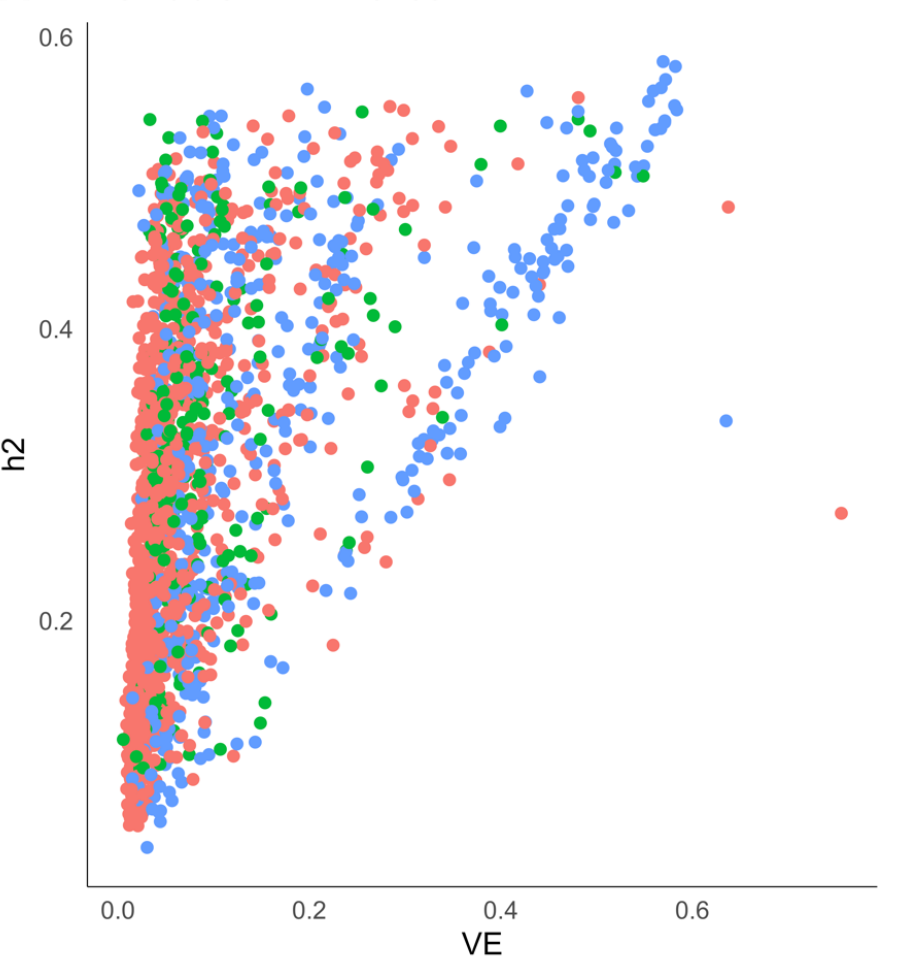

B ESS DNAm sites

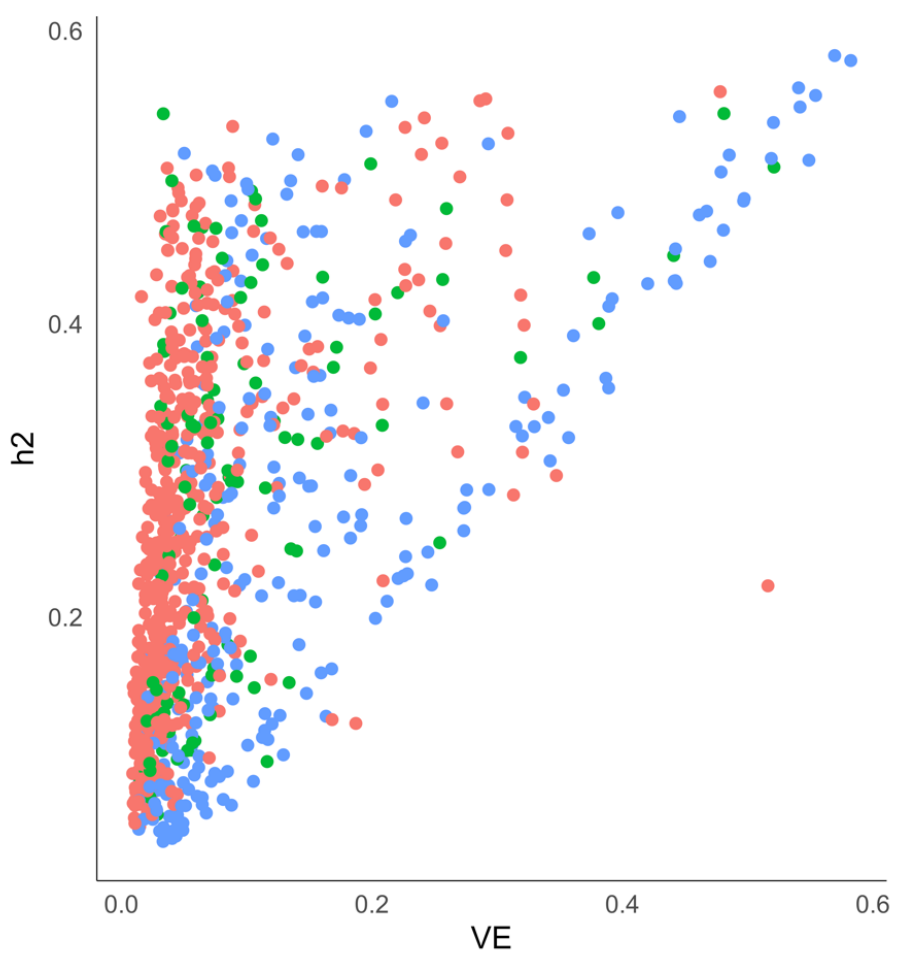

C PhenoAge DNAm sites

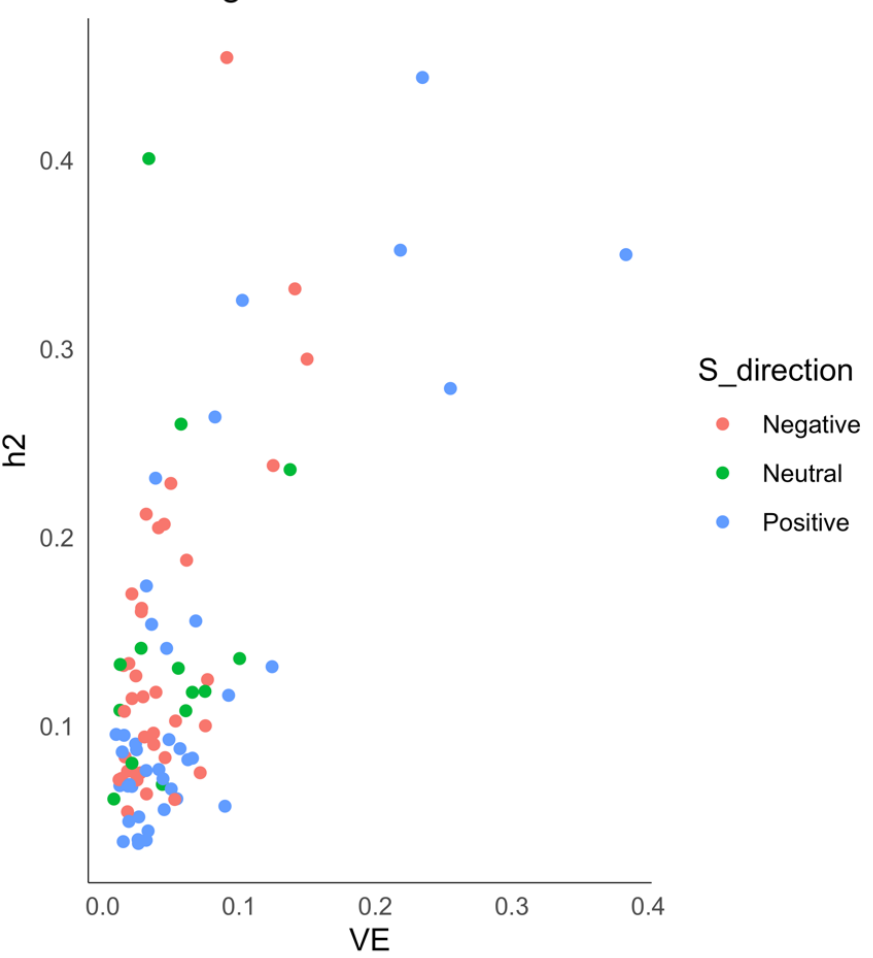

Figure 3 | Relationship between $\mathbf{h}^{2}$ SNP calculated by BayesNS and mean variance explained (VE). For (A) 1804 highly heritable DNAm sites, (B) 887 ESS DNAm sites and (C) 74 PhenoAge DNAm sites. Estimates of $S$ coloured: red (negative $<=-0.1$ ), green (neutral $-0.1-0.1$ ) and blue (positive $>0.1$ ). 
A Positive Highly Heritable

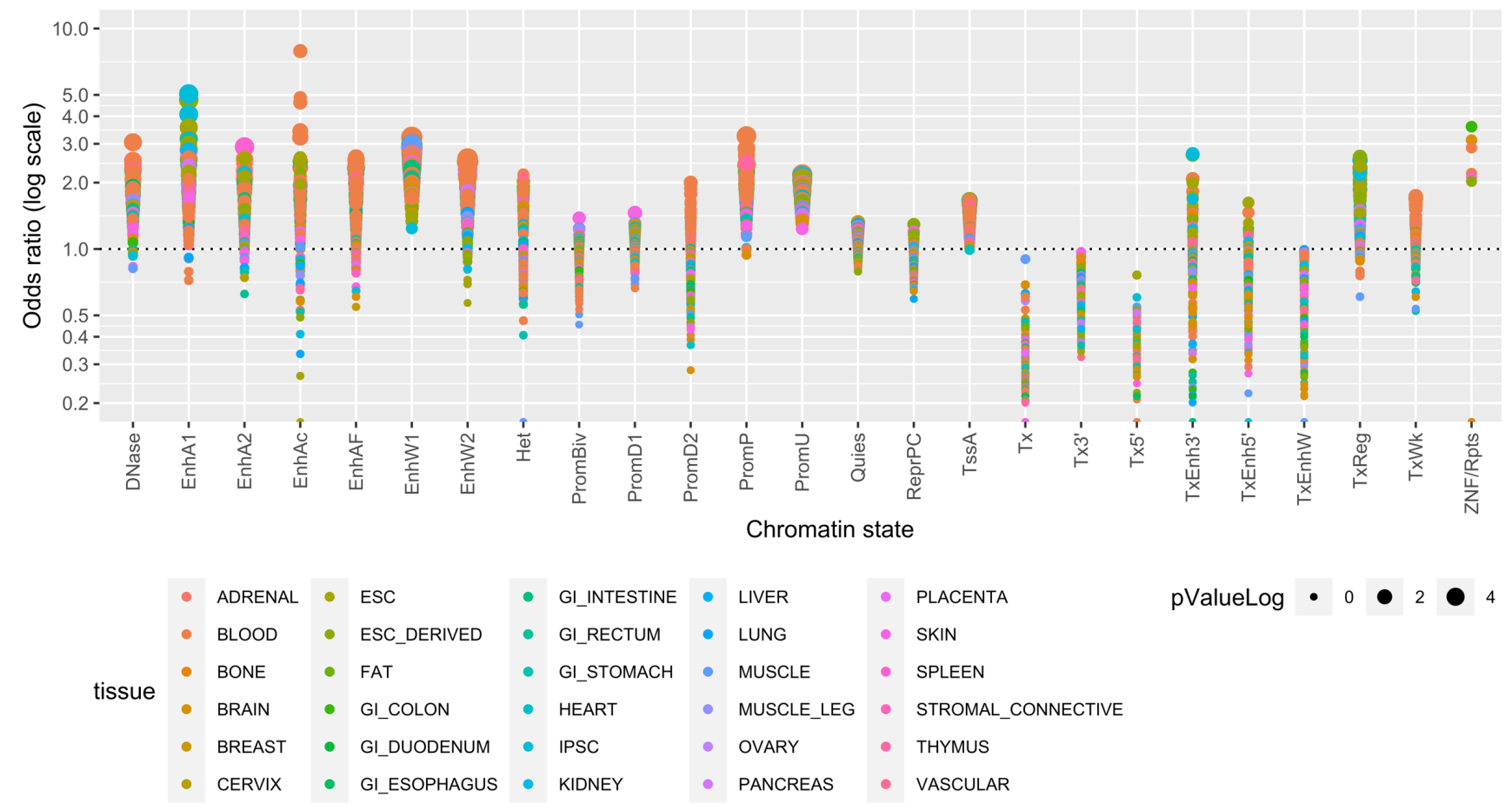

B Negative Highly Heritable

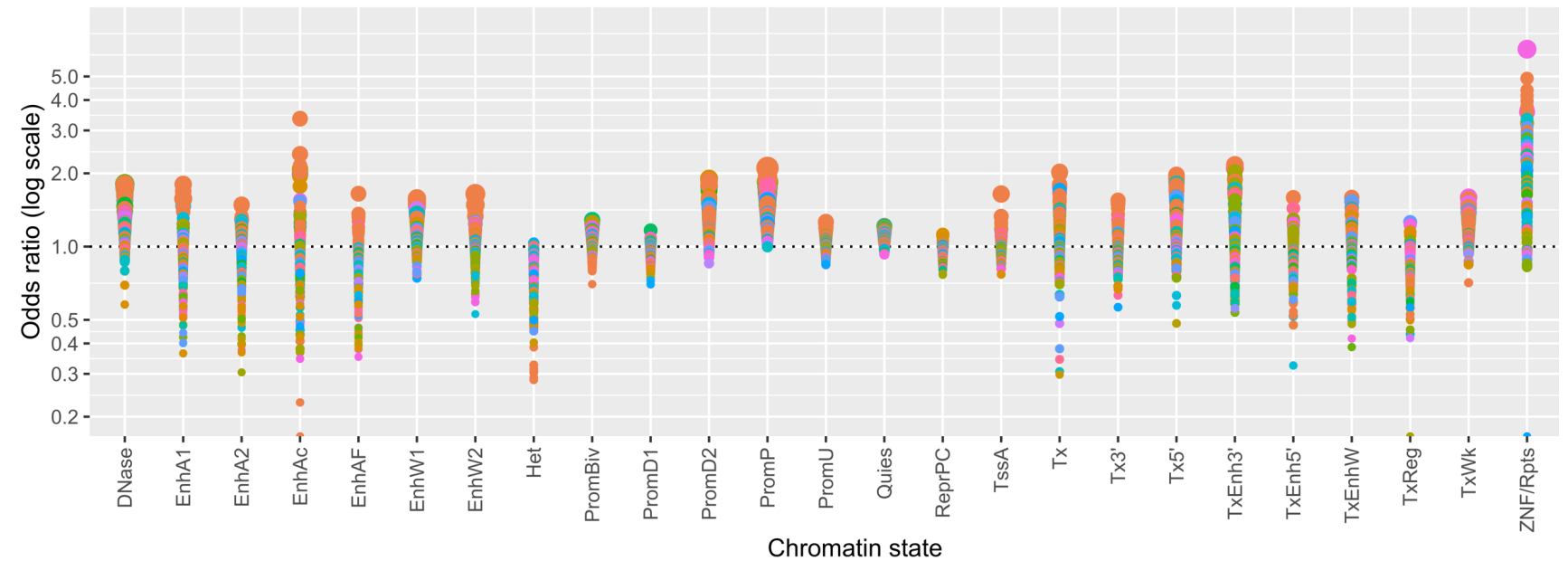

Figure 4 | Enrichment or depletion of DNAm sites in predicted chromatin states for DNAm sites from the highly heritable probe set with estimates of $S>=0.5$ (positive highly heritable) and $S<=-0.5$ (negative highly heritable). Odds ratio (on log scale) shown on the $y$ axis and chromatin state on the $x$ axis. Size of circle represents the $-\log 10 P$ value. Enrichment analysis performed via two-sided Fisher's exact test implemented in LOLA ${ }^{1} .25$ chromatin states abbreviations: TssA, Active TSS; PromU, Promoter Upstream TSS; PromD1, Promoter Downstream TSS with DNase; PromD2, Promoter Downstream TSS; Tx5', Transcription 5'; Tx, Transcription; Tx3', Transcription 3'; TxWk, Weak transcription; TxReg, Transcription Regulatory; TxEnh5', Transcription 5' Enhancer; TxEnh3', Transcription 3' Enhancer; TxEnhW, Transcription Weak Enhancer; EnhA1, Active Enhancer 1; EnhA2, Active Enhancer 2; EnhAF, Active Enhancer Flank; EnhW1, Weak Enhancer 1; EnhW2, Weak Enhancer 2; EnhAc, Enhancer Acetylation Only; DNase, DNase only; ZNF/Rpts, ZNF genes \& repeats; Het, Heterochromatin; PromP, Poised Promoter; PromBiv, Bivalent Promoter; ReprPC, Repressed PolyComb, Quies, Quiescent/Low. 


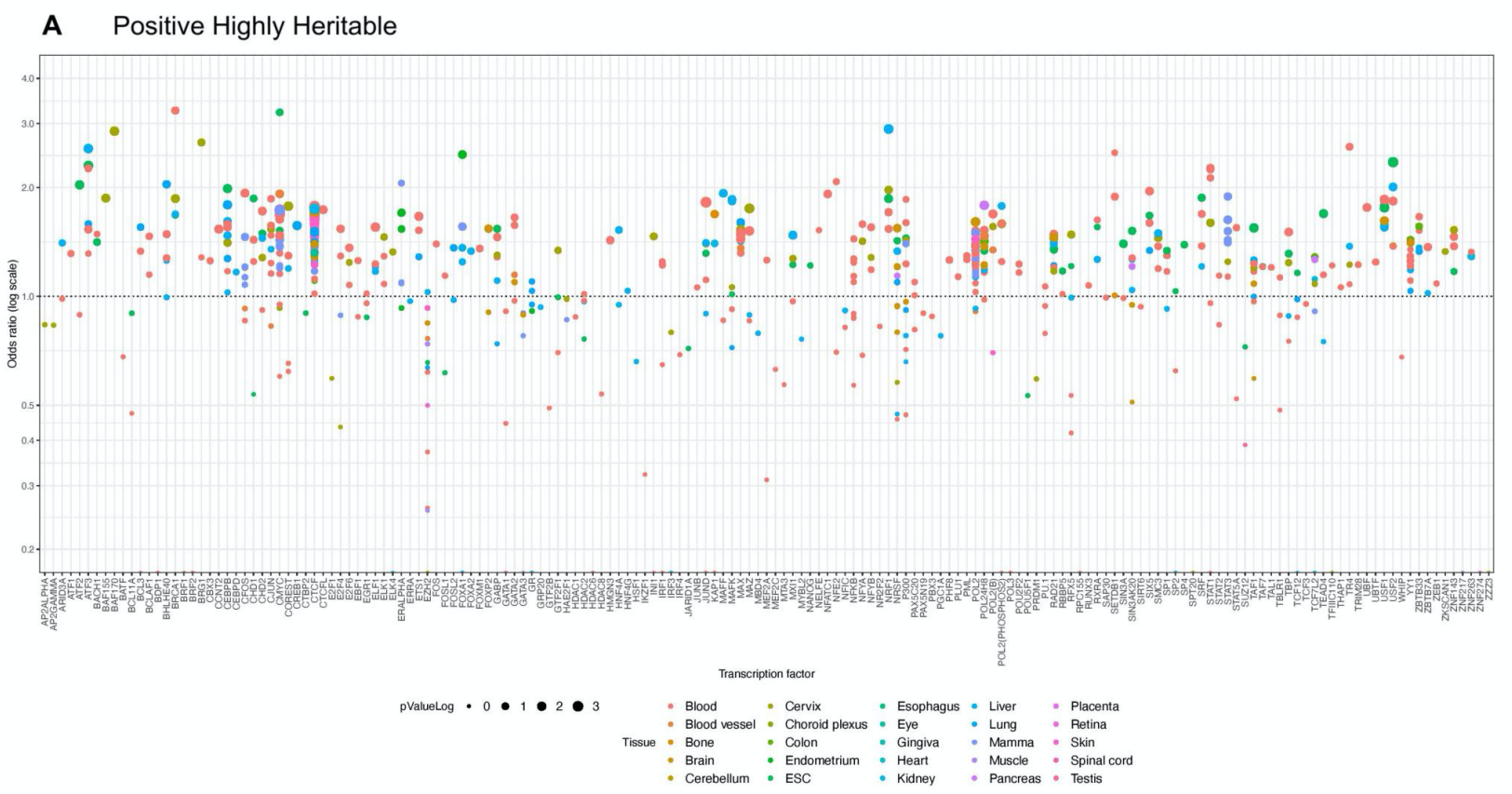

B Negative Highly Heritable

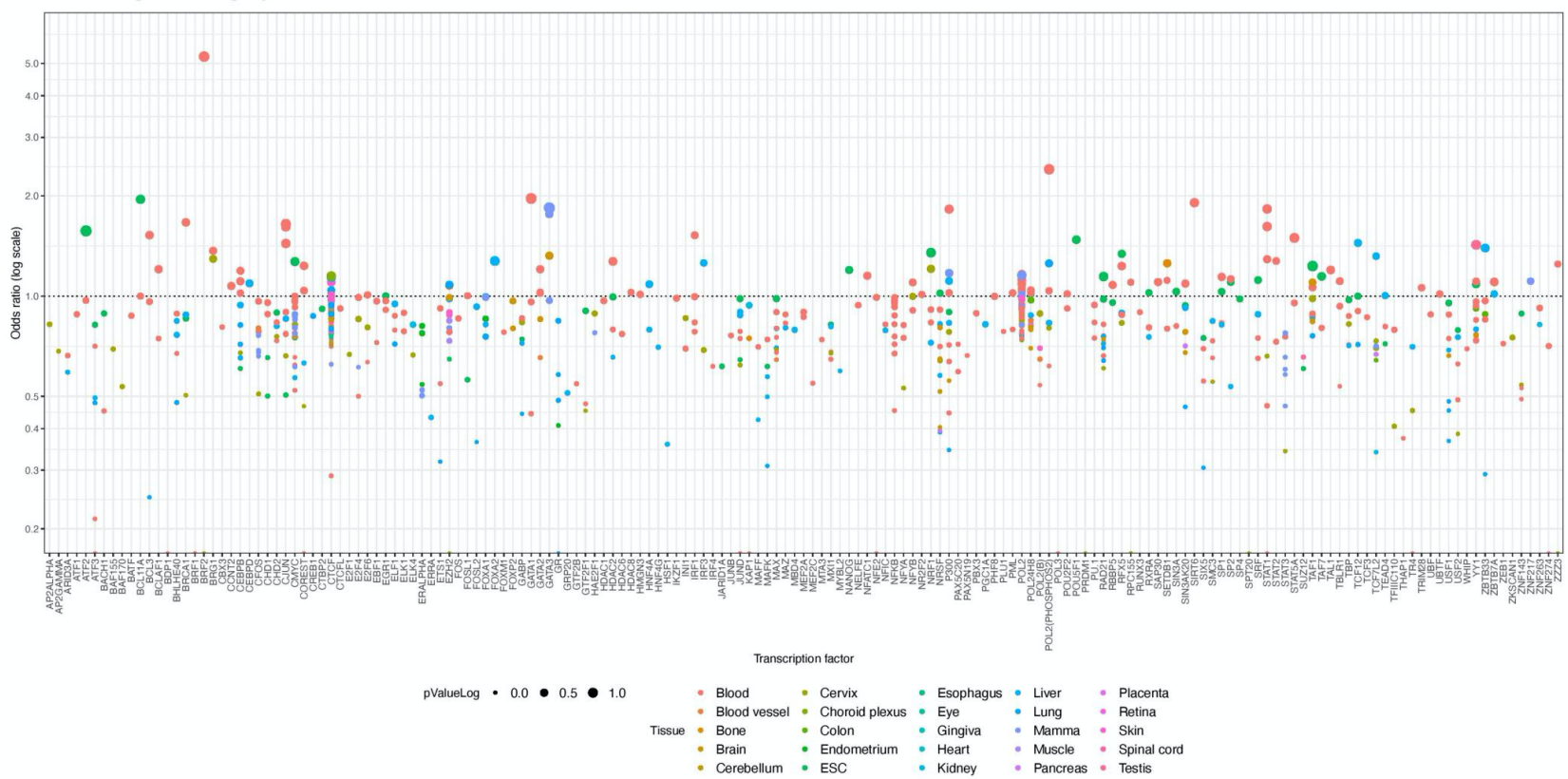

Figure 5 | Enrichment or depletion of DNAm sites in transcription factors (TFs) for DNAm sites from the highly heritable probe set with estimates of $S>=0.5$ (positive highly heritable) and $S<=-0.5$ (negative highly heritable). Odds ratio (on log scale) shown on the $y$ axis and chromatin state on the $x$ axis. Size of circle represents the $-\log 10 P$ value. Enrichment analysis performed via two-sided Fisher's exact test implemented in LOLA 1 . 


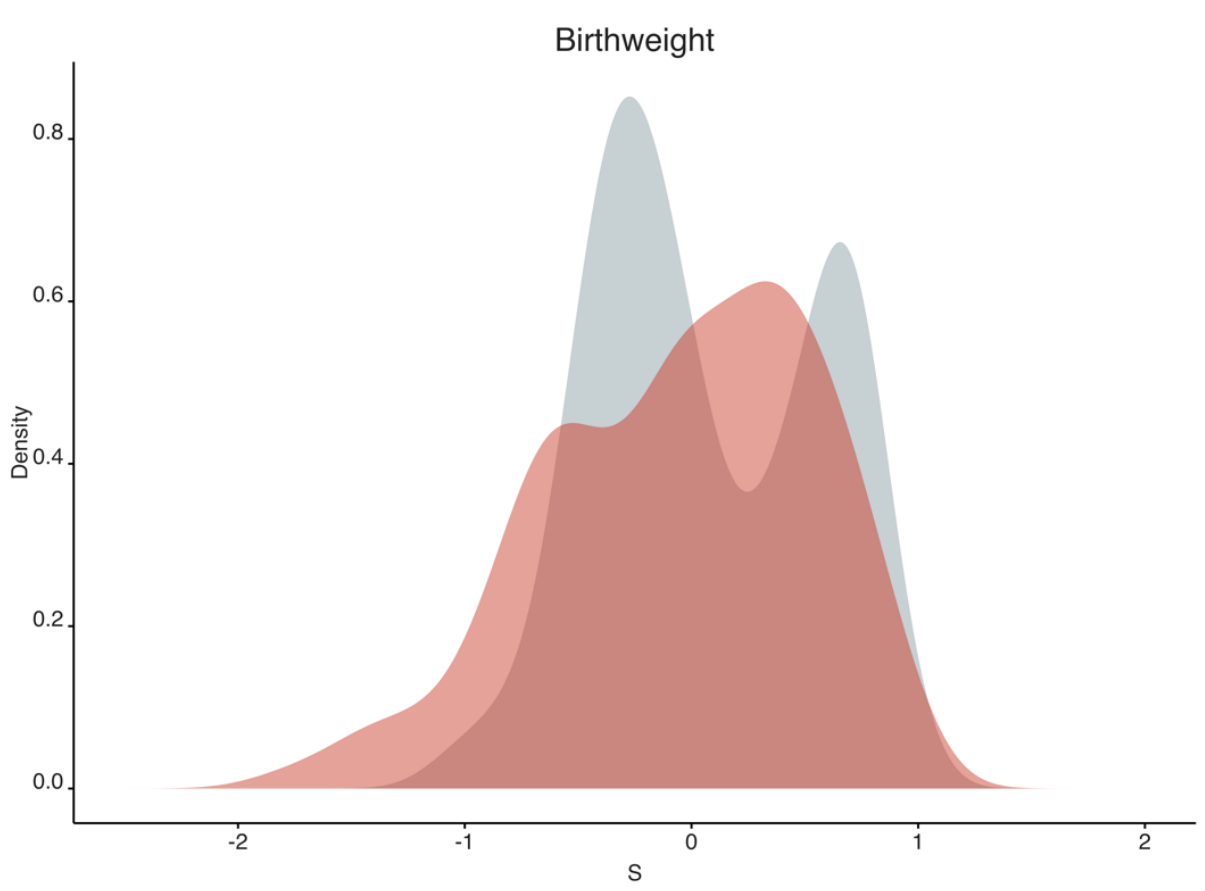

CpG background birthweight

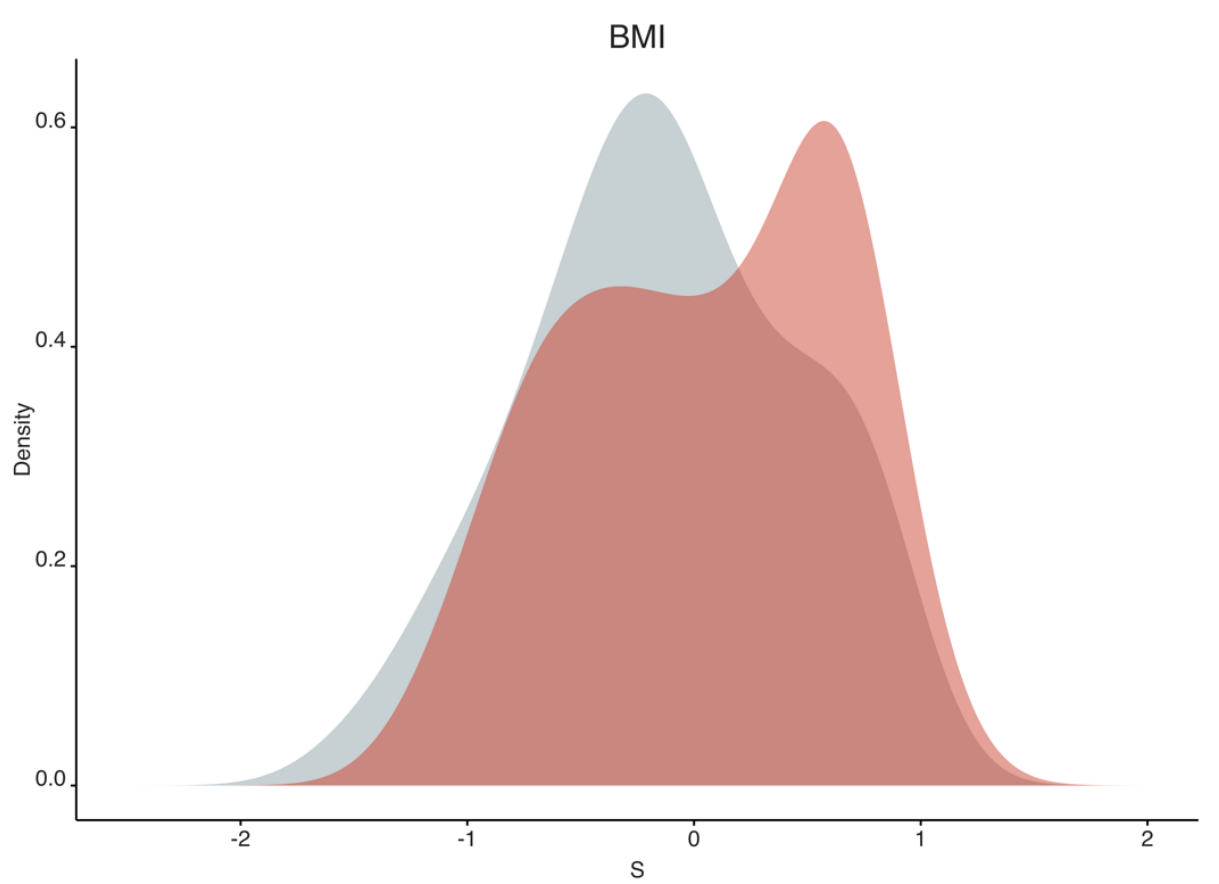

$\mathrm{CpG}$ background $\square \mathrm{BMI}$

Figure 6 | Distribution of estimates of $S$ for DNAm sites associated with birthweight and BMI compared to background DNAm sites. $S$ is estimated using the relationship between SNP effect size and MAF, when $S=0$ SNP effect size is independent of MAF (neutral), $S>0$ indicates positive selection, $S<0$ indicates negative selection. Birthweight and BMI associated DNAm sites shown in red and matched DNAm sites (matched on GC/CpG content and heritability) shown in grey. 
bioRxiv preprint doi: https://doi.org/10.1101/2021.11.25.469994; this version posted November $25,2021$. The copyright holder for this preprint (which was not certified by peer review) is the author/funder, who has granted bioRxiv a license to display the preprint in perpetuity. It is made available under aCC-BY 4.0 International license.

\section{References}

1. Sheffield NC, Bock C. LOLA: enrichment analysis for genomic region sets and regulatory elements in R and Bioconductor. Bioinformatics. 2016;32(4):587-589. 
bioRxiv preprint doi: https://doi.org/10.1101/2021.11.25.469994; this version posted November 25, 2021. The copyright holder for this

preprint (which was not certified by peer review) is the author/funder, who has granted bioRxiv a license to display the preprint in perpetuity. It is made available under aCC-BY 4.0 International license. 
bioRxiv preprint doi: https://doi.org/10.1101/2021.11.25.469994; this version posted November 25, 2021. The copyright holder for this

preprint (which was not certified by peer review) is the author/funder, who has granted bioRxiv a license to display the preprint in perpetuity. It is made available under aCC-BY 4.0 International license. 